\title{
Dynamic Changes in Occupancy of Histone Variant H2A.Z during Induced Somatic Cell Reprogramming
}

\author{
Fulu Dong, ${ }^{1,2}$ Zhenwei Song, ${ }^{3}$ Jiali Yu, ${ }^{2}$ Baole Zhang, ${ }^{1}$ BaoChun Jiang, ${ }^{1}$ Ying Shen, ${ }^{4}$ \\ Youde Lu, ${ }^{1}$ Chunlei Song, ${ }^{1}$ Peiqing Cong, ${ }^{5}$ and Honglin Liu ${ }^{1}$ \\ ${ }^{1}$ Department of Animal Breeding \& Genetics, College of Animal Science \& Technology, Nanjing Agricultural University, \\ Nanjing 210095, China \\ ${ }^{2}$ Institutes of Biology and Medical Sciences, Soochow University, Suzhou 215123, China \\ ${ }^{3}$ Medical College, Hunan Normal University, Changsha 410006, China \\ ${ }_{4}^{4}$ Jinling Hospital, Medical School of Nanjing University, Nanjing 210093, China \\ ${ }^{5}$ State Key Laboratory of Biocontrol, Sun Yat-sen University, Guangzhou 510275, China
}

Correspondence should be addressed to Honglin Liu; liuhonglin@njau.edu.cn

Received 8 April 2015; Accepted 25 August 2015

Academic Editor: Aster H. Juan

Copyright (C) 2016 Fulu Dong et al. This is an open access article distributed under the Creative Commons Attribution License, which permits unrestricted use, distribution, and reproduction in any medium, provided the original work is properly cited.

The development of induced pluripotent stem cells (iPSCs) has enabled study of the mechanisms underlying cellular reprogramming. Here, we have studied the dynamic distribution of H2A.Z during induced reprogramming with chromatin immunoprecipitation deep sequencing (ChIP-Seq). We found that H2A.Z tended to accumulate around transcription start site (TSS) and incorporate in genes with a high transcriptional activity. GO analysis with H2A.Z incorporated genes indicated that most genes are involved in chromatin assembly or disassembly and chromatin modification both in MEF and Day 7 samples, not in iPSCs. Furthermore, we detected the highest level of incorporation of H2A.Z around TSS in Day 7 samples compared to MEFs and iPSCs. GO analysis with only incorporated genes in Day 7 also displayed the function of chromatin remodeling. So, we speculate H2A.Z may be responsible for chromatin remodeling to enhance the access of transcription factors to genes important for pluripotency. This study therefore provides a deeper understanding of the mechanisms underlying induced reprogramming.

\section{Introduction}

The development of induced pluripotent stem cell (iPSC) technology that was first reported in 2006 by Takahashi and Yamanaka [1] has facilitated great advancements in the field of stem cell biology. The ability to reprogram somatic cells to a pluripotent state has enhanced our understanding of the mechanisms underlying nuclear reprogramming and the regulation of cell stemness and spawned new approaches for regenerative medicine and disease therapy $[2,3]$. However, while iPSC technology offers unprecedented opportunities, the precise molecular steps by which donor cells regain pluripotency remain somewhat unexplained [4]. Furthermore, a number of issues [5] surrounding the efficiency and stability of the induced reprogramming process need to be resolved.
Some studies have provided insights into changes in gene expression patterns during reprogramming [6-8]. However, differences between donor cells and iPSCs, and embryonic stem cells (ESCs) and iPSCs exist at the epigenetic level as well as in gene expression $[9,10]$. Dynamic chromatin structures affect transcriptional patterns by altering the accessibility of transcription factors or other regulatory proteins to DNA through permissive remodeling machinery. The unique epigenetic state of the ESC is characterized as a higher order chromatin structure [12], suggesting that the hyperdynamic plasticity of chromatin proteins may be responsible for maintaining pluripotency. Furthermore, both remodeling complexes and histone variation can affect chromatin structure and cell fate. For example, brahma-associated factor (BAF) complexes can contribute to stable, tissue-specific memory of cell fate $[11,13]$, and histone variant replacement can regulate 
TABLE 1: Clean data output from H2A.Z ChIP-Sequence.

\begin{tabular}{lccc}
\hline Sample ID & Length of read (bp) & Total number of reads & Output (bp) \\
\hline MEFs & 49 & $12,807,843$ & $627,584,307$ \\
Day 7 cells & 49 & $12,910,314$ & $632,605,386$ \\
iPSCs & 49 & $12,779,646$ & $625,908,654$ \\
\hline
\end{tabular}

TABLE 2: Mapped reads output from H2A.Z ChIP-Sequence.

\begin{tabular}{lccccc}
\hline \multirow{2}{*}{ Sample ID } & Total reads & Reads & Mapped & \multicolumn{2}{c}{ Unique mapped } \\
& & Percentage & $97.27 \%$ & $11,216,366$ & $87.57 \%$ \\
\hline MEFs & $12,807,843$ & $12,458,074$ & $97.41 \%$ & $10,961,715$ & $84.91 \%$ \\
Day 7 cells & $12,910,314$ & $12,575,751$ & $97.17 \%$ & $10,577,813$ & $82.81 \%$ \\
iPSCs & $12,779,646$ & $12,412,347$ & Percentage \\
\hline
\end{tabular}

stem cell differentiation through critically determining the gene expression profile [14]. However, whether a dynamic chromatin state leads to changes in cell fate during iPSC development remains unclear.

The incorporation of specific histone variants underlies changes in chromatin structure which are crucial for transcriptional control during remodeling. One of the most highly evolutionarily conserved histone variants is $\mathrm{H} 2 \mathrm{~A} . \mathrm{Z}$, and this variant is responsible for unique structural features of chromatin. H2A.Z possesses a tetramer-dimer docking domain [15], is usually enriched at the transcriptional start site (TSS) of genes, and plays critical roles in gene regulation through transcriptional activation $[4,16]$. H2A.Z has also been implicated in chromatin regulation processes as well as in the establishment of chromatin boundaries for nucleosome exchange and polycomb repression [4, 17]. However, the relationship between these seemingly disparate functions and induced reprogramming of pluripotent cells remains obscure. Understanding exactly how different histone variants influence gene expression patterns and ultimately cell fate will enhance our understanding of induced reprogramming.

To this end, we explored the H2A.Z genome-wide deposition profile of three cell samples representing different stages of reprogramming from murine embryonic fibroblasts (MEFs) to iPSCs using chromatin immunoprecipitation (ChIP) coupled with high-throughput sequencing (ChIPSeq). We identified drastic enrichment of H2A.Z deposition in the transition period from Day 7 to characterization as an iPSC. Examination of H2A.Z dynamics during this transition period has enhanced our understanding of the roles of histone variants in facilitating induced reprogramming. Our results find that $\mathrm{H} 2 \mathrm{~A} . \mathrm{Z}$ may contribute to the phenotypic plasticity of chromatin structure, thereby enhancing transcription factor access to DNA and contributing to the reprogrammed cell state.

\section{Results}

2.1. Dynamic Occupancy of H2A.Z Variant during iPSC Development. To investigate whether the histone variant $\mathrm{H} 2 \mathrm{~A} . \mathrm{Z}$ is involved in reprogramming from MEFs to iPSCs, we utilized ChIP-Seq technology to examine the dynamics of chromatinbound $\mathrm{H} 2 \mathrm{~A} . \mathrm{Z}$ in the process of induced reprogramming.
Our previous report showed mouse iPSCs (m-iPSCs) were successfully generated under ectopic expression of Oct3/4, Sox2, and Klf4 (OSK) in the presence of Vc [18]. Importantly, there were aggregated cells that appeared at Day 7 and always became the iPSC clone in the end. Here, the initial cell MEF, Day 7 cells (MEFs were induced for 7 days, and aggregated cells were used in subsequent analysis), and iPSCs (clones from MEF undergoing induction for 14 days) representing different stages of the reprogramming course were collected to fulfill the ChIP-Seq.

To avoid the false positive data due to nonspecificity from the H2A.Z antibody, we checked the antibody with an ordinary experiment fulfilled by IgG antibody ChIP. The DNA deposited with H2A.Z antibody fulfilled by ChIP was quantized by qRT-PCR. Special affinity genes hoxall, hoxb1, zfpm2, lrx2, pax5, neurog1, and hoxb2 selected from UCSC database and report [19] were immunoprecipitated with H2A.Z and IgG antibody independently and then tested by qRT-PCR with the same primers (Figure S1 in Supplementary Material available online at http://dx.doi.org/10.1155/2015/3162363).

We achieved over $20 \mathrm{Mb}$ output clean dataset per cell sample (Table 1). Reads were aligned to a reference genome that combines mouse genome mm9. Using SOAP 2.21 software, we selected effectively mapped reads as detailed in Table 2. Further selection of reads that were mapped to unique genomic regions based on the precise mapping annotations "promoter," "UTR," "CDS," and "intergenic" refined the dataset (Table 2). Employing MACS 1.4.0 [20] software, we identified $23,818,13,744$, and 14,768 peaks among the MEF, Day 7 , and iPSC cell samples, respectively (Table 3). Peaks are preferentially dropped in intergenic, promoter, intron, and $5^{\prime}$ UTR regions both in MEF and iPSC cell samples (Figures 1(a) and 1(c)). Distinctively, 31.55\% of the peaks were found on promoters in Day 7 cell samples more than in intergenic regions (23.64\%) and any other genomic regions. Certainly, the percentage of peaks on promoter regions in Day 7 (31.55\%) was higher than MEF (26.55\%) and iPSC (25.04\%).

We know that different genetic regions contain a different number of bases. In order to eliminate the influence of nonspecific incorporation, we evaluated the relative enrichment of peaks in genetic elements depending on ratio 
TABLE 3: Output of peaks from H2A.Z ChIP-Sequence.

\begin{tabular}{|c|c|c|c|c|c|}
\hline \multirow{2}{*}{ Sample ID } & \multirow{2}{*}{ Total number of peaks } & \multicolumn{3}{|c|}{ Length of peaks (bp) } & \multirow{2}{*}{ Percentage } \\
\hline & & Total & Mean & Median & \\
\hline MEFs & 23,818 & $32,163,617$ & 1,350 & 1,581 & $1.18 \%$ \\
\hline Day 7 cells & 13,744 & $17,951,826$ & 1,306 & 1,516 & $0.66 \%$ \\
\hline iPSCs & 14,768 & $12,532,924$ & 849 & 942 & $0.46 \%$ \\
\hline
\end{tabular}

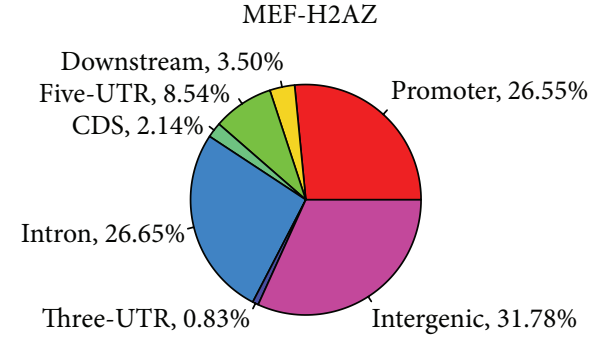

(a)

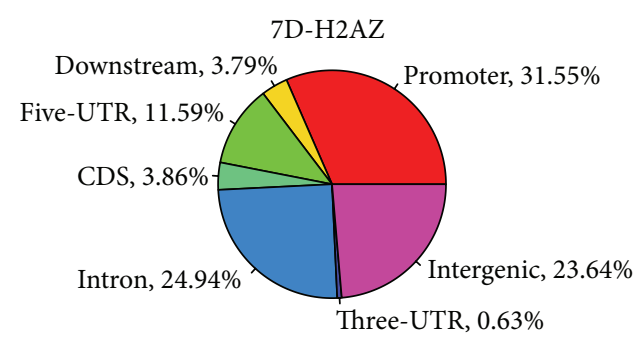

(b)

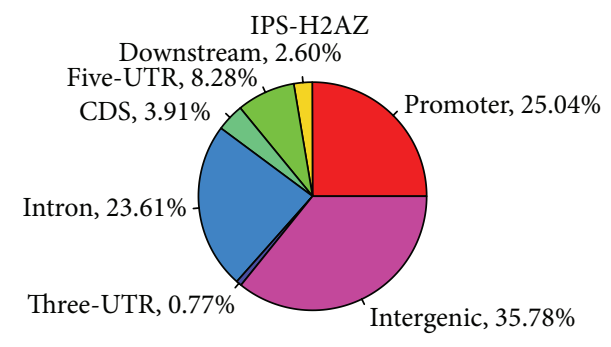

(c)

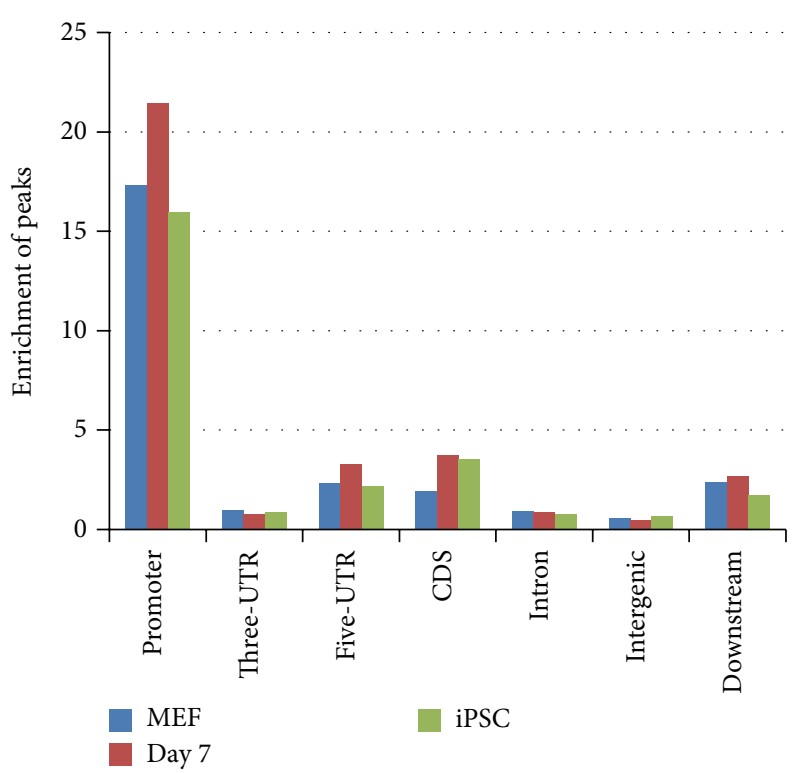

(d)

FIGURE 1: Distribution and enrichment of H2A.Z in different gene regions. (a-c) Mapping of peaks representing H2A.Z occupancy on a genome-wide scale relative to RefSeq mouse genes. "Promoter" and “downstream” are defined as $2 \mathrm{~kb}$ of $5^{\prime}$ or $3^{\prime}$ flanking regions. The "intergenic" region refers to all locations other than the promoter, $5^{\prime} \mathrm{UTR}$, CDS, intron, $3^{\prime} \mathrm{UTR}$, or "downstream" (d). Relative enrichment of H2A.Z peaks in gene elements defined as (number of ChIP sequencing peaks in one region/total number of ChIP sequencing peaks in genome)/(number of whole genome bases in one region/total number of whole genome bases).

[(number of ChIP sequencing peaks in one region/total number of ChIP sequencing peaks in genome)/(number of whole genome bases in one region/total number of whole genome bases)]. Figure 1(d) demonstrates that most peaks specifically were mapped to promoters preferentially, whereas small sets of peaks were mapped in regions including "downstream," $5^{\prime} \mathrm{UTR}$, and CDS while some peaks were mapped to others, including intron, $3^{\prime} \mathrm{UTR}$, and intergenic regions. Interestingly, H2A.Z occupied more DNA sequences in the promoter during the period of development from MEF to Day 7 but departed from these sites with iPSC formation (Figure 1(d)).
2.2. H2A.Z Is Deposited at the TSS Region during Induced Reprogramming. To deeply investigate the deposition of H2A.Z at promoter regions across the entire genome, we compared the presence of H2A.Z at annotated gene regions $5 \mathrm{~kb}$ upstream and $5 \mathrm{~kb}$ downstream TSSs with normalized reads in each of the three cell types. We found that H2A.Z was enriched on both sides of the TSS, with a small drop over the TSS site (Figure 2(a)). However, the average normalized reads have unmarked changes near the transcriptional end sites (TESs) (Figure 2(b)). These double peak curves and the relatively depleted distribution style of H2A.Z are in agreement with another report [21, 22], which proved that the result 


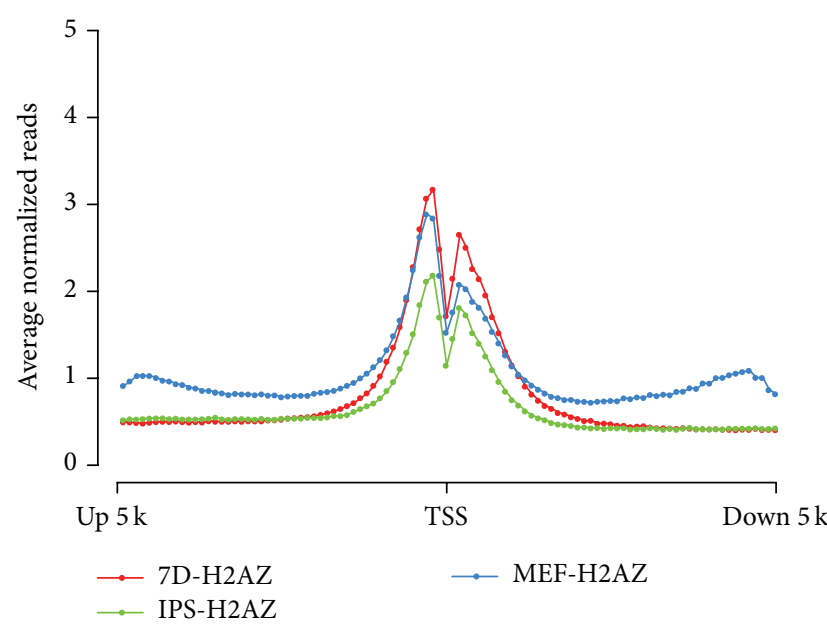

(a)

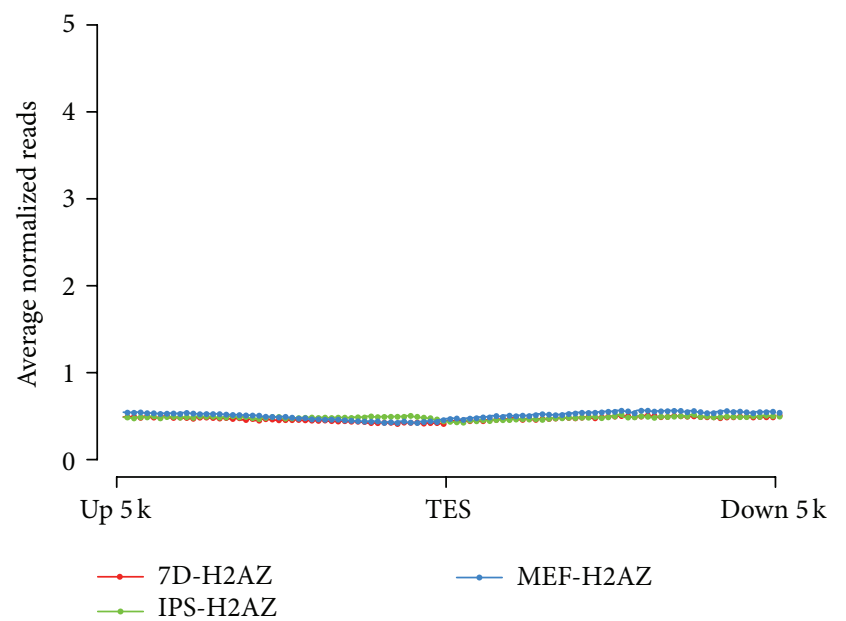

(b)

FIGURE 2: The distribution of average normalized reads in TSS and TES gene regions. The regions $5 \mathrm{~kb}$ upstream and downstream the TSS (a) and TES (b) are divided into 40 windows, and H2A.Z average normalized reads coverage is assessed in each window.

is believable and indicated H2A.Z preferentially deposits upstream TSSs. Consistent with the above result, Day 7 cells have the highest average level of H2A.Z occupancy, while the iPSCs have the lowest.

In mRNA expression level, such immune response genes (gene expression level: the highest at Day 7 compared to MEF and iPSCs) were noticeable [18]. However, gene related to acetylation, chromatin assemblies, and cell cycle was deposited with higher H2A.Z occupancy (Figure S2). These data indicate that cells induced by infection by OSK viral vectors change their cell fate through the help with H2A.Z's accessing to the epigenetics factors.

To examine the relationship between the enrichment of H2A.Z on chromatin and gene expression levels, we compared H2A.Z occupancy and the gene expression data from our previous report [18] in different genes. We then separated genes into four classes based on expression levels and displayed H2A.Z levels around TSSs. This revealed that, for all three samples, H2A.Z displayed high occupancy flanking TSSs in high expression and middle expression genes and decreases with decreasing transcription genes (Figure 3). This suggested a correlation between the incorporation of H2A.Z around TSS and gene expression in the development of iPSC.

Noticeably, transcription factors Oct4, Nanog, Klf4, cMyc, sox2, and Lin28 were usually employed to fulfill the induced reprogramming. In their DNA sequence, the enrichment of H2A.Z was dynamic and complex (Figure S3). However, the data showed that there was the highest occupancy of H2A.Z in the upstream region of Nanog and Lin28 individually. And on the wide whole sequence, the peak was shifted from downstream to upstream during the induced reprogramming. As the expression level of pluripotent genes was upregulated in the process of induced reprogramming, it indicates that the H2A.Z benefits the reactivation of these genes by the dynamic occupancy. Likely, the enrichment of H2A.Z on cMyc was dynamically shifted from upstream to TSS. Strikingly, the occupancy kept a higher level in these regions during the whole induced reprogramming. For Nanog and Oct4, the peaks always appeared in the CDS regions and more deposited H2A.Z at Day 7. This data supports that binding between Oct4 and H2A.Z downstream [23]. Unlike these transcriptional factors, the enrichment on Sox 2 did not have the highest level on the TSS region at Day 7; however, there were continues occupancy both at the TSS and TES regions at Day 7. It indicted that regulation of these pluripotent genes' expression by H2A.Z may rely on the different behavioral style of dynamic occupancy.

\subsection{H2A.Z Occupancy Contributes to Induced Reprogram-} ming through Chromatin Remodeling. H2A.Z nucleosome composition at promoters and gene body influences nucleosome stability and transcriptional state [16], and the above results have indicated that H2A.Z preference incorporation to the high expressed genes around TSS during somatic cell reprogramming (Figure 3); we speculate the incorporation of H2A.Z can regulate the expression of binding genes and then promote reprogramming. To investigate the function of H2A.Z occupancy during induced somatic cell reprogramming, we selected genes which detected H2A.Z deposition in the promoter or gene body regions in three cell samples (Tables S1-S3). We identified 12281 genes that were occupied by H2A.Z in MEFs, 9598 genes in Day 7 cells, and 7944 genes in iPSCs. By biological processes analysis performed on H2A.Z related genes, there were 116 biological processes in iPSCs, 168 in Day 7 cells, and 237 in MEFs (Tables S4-S6). These genes which H2A.Z occupied in MEFs were more various in gene ontology than both Day 7 cells and iPSCs. It indicated that H2A.Z deposited more widely at the first time and eventually located on limited scale. For this biological processes analysis, the terms gene expression, regulation of gene expression, transcription, and regulation of transcription ranked much higher in iPSCs than in Day 7 cells and MEFs. None of the genes occupied by H2A.Z were involved in chromatin modification, assembly, or disassembly in iPSCs, but genes involved in 


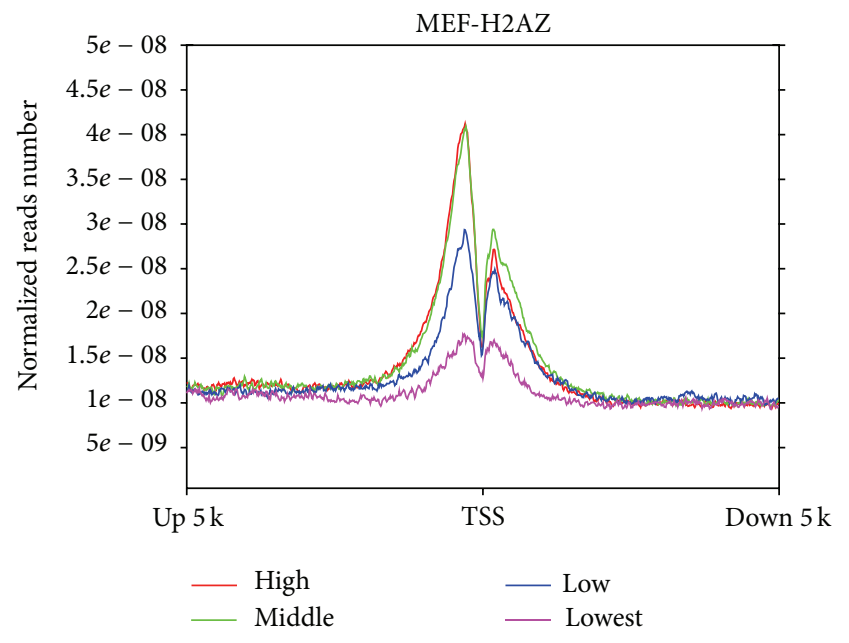

(a)

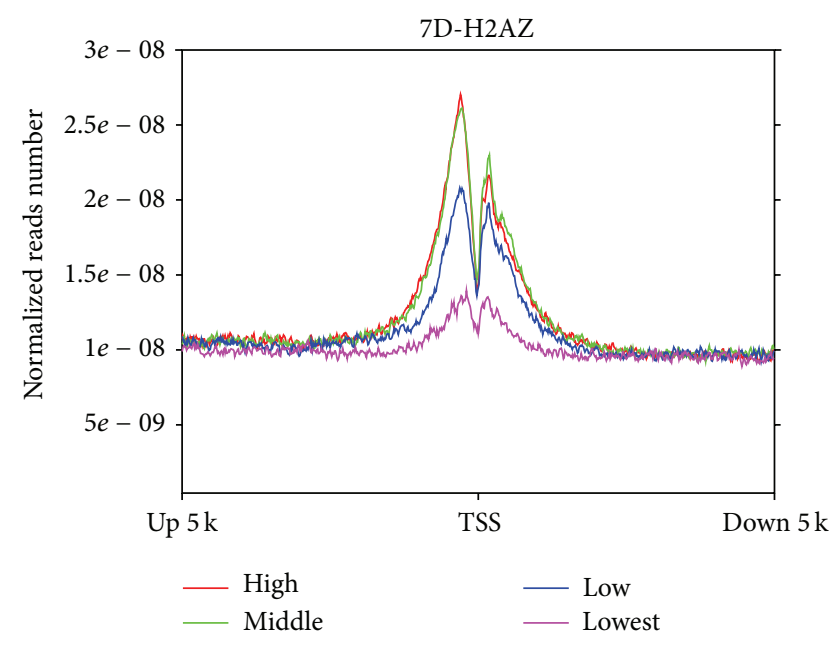

(b)

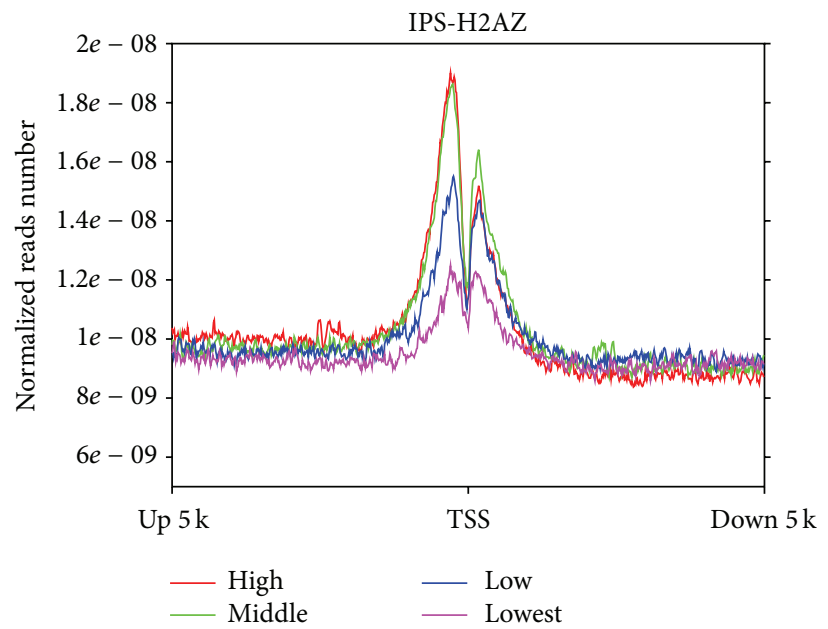

(c)

Figure 3: Comparison of microarray expression data and enrichment of H2A.Z in MEFs, Day 7 cells, and iPSCs. Genes are aligned at the TSS and divided into four parts (high, middle, low, and lowest) by decreasing expression level. Statistical H2A.Z normalized reads number in MEFs (a), Day 7 cells (b), and iPSCs (c).

these processes were occupied by H2A.Z in Day 7 cells and MEFs (Figure 4).

Most genes that were occupied by H2A.Z in all three cell types are involved in metabolic processes. While the same biological processes were largely present in the top 10 across the cell types, the label "gene expression" was present here in Day 7 cells. This indicates that genes occupied by H2A.Z at Day 7 are more involved in gene expression (Figure 5). Further analysis found that 2200 genes were occupied only in MEFs, 478 genes only in Day 7 cells, and 1067 genes only in iPSCs (Figure 6(a)). H2A.Z enhances specific incorporation in Day 7 (Figures 1 and 2). So, we speculate that the increased enrichment of H2A.Z in Day 7 may change the expression of specific genes and then promote reprogramming. Biological processes analysis was performed with 478 genes with H2A.Z incorporation only in Day 7 cells. We found they were primarily involved in macromolecular complex assembly and organization, including DNA packaging, chromatin assembly or disassembly, and protein-DNA complex assembly
(Figure 6(b)). This indicated that H2A.Z tended to occupy specific genes, being especially involved in the regulation of epigenetic factor genes that affect chromatin structure after Day 7.

\section{Discussion}

H2A.Z is evolutionarily conserved in both molecular sequence and structure [24], altering chromatin structure through its deposition at DNA binding sites. In addition to the alternative chromatin structure induced by binding of histone variant H2A.Z, the access of other regulatory elements can be regulated by the kinetics status of the nucleosome. Our data have demonstrated that histone variant $\mathrm{H} 2 \mathrm{~A} . \mathrm{Z}$ is always present in the promoter of active genes during induced somatic cell reprogramming (ISCR) (Figure 3). Recent reports [25] support the idea that H2A.Z can deposit independently of replication, and it is preferentially enriched in active gene promoters. And 


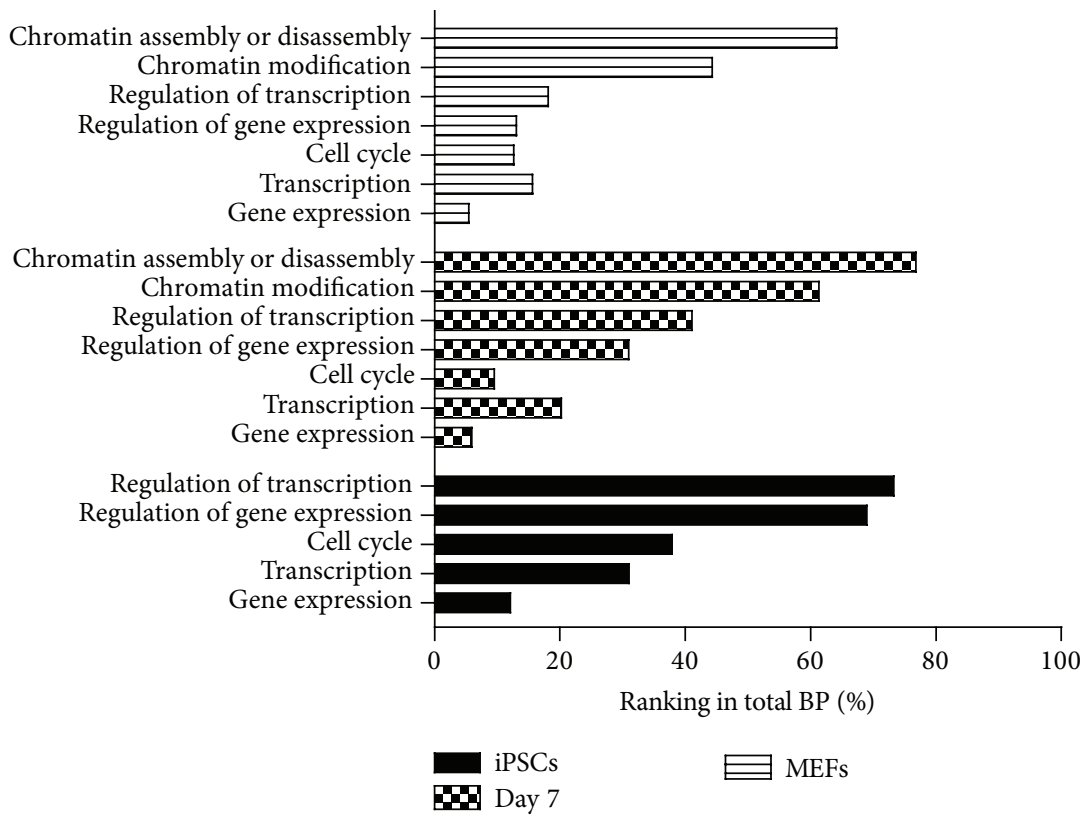

FIGURE 4: Rank of the same biological process in different samples. Ranking depicts biological processes in order of changes to expression of genes in which H2A.Z was incorporated. The total number of biological processes in distinct samples was normalized as 100 , and the enrichment of selected processes is presented as a percentage. The shorter bar indicates the higher order in GO categories, which means higher activity genes.

this access of TSS may be responsible for the feature of active genes [26] and offer the inactivated gene a feasible nucleosome with active promoters [27]. Previously reported data show that active genes were highly occupied by H2A.Z, which face-lifted self-renewal of stem cells [23]. This is consistent with a role for $\mathrm{H} 2 \mathrm{~A} . \mathrm{Z}$ in facilitating the generation of iPSCs through its deposition at promoters across the genome during ISCR.

H2A.Z is believed to facilitate the assembly or disassembly of chromatin and to increase transcriptional activity. In yeast, H2A.Z is often located at inactive promoters within nucleosomes where it aids in providing the correct architecture for transcription activation [28]. Our data have shown that many H2A.Z occupied genes are involved in biological processes related to gene expression in all three of the cell samples examined. Previous reports have established that acetylated $\mathrm{H} 2 \mathrm{~A}$ is often present in active nucleosomes [29] and that these acetylated proteins can recruit protein complexes for regulation of gene transcription [30]. It is apparent that genes occupied by H2A.Z can be easily marked as active and this combination between H2a.z and DNA regions may be signals for recruitment of reassemble acetyl enzyme complexes. Furthermore, H2A.Z is a coactivator required for the complete acetylation of histones, which facilitates access to gene promoters for transcription factors [30]. Our data indicate that H2A.Z may have a strong influence on the regulation of gene expression through its deposition at TSSs during ISCR. It strongly supports the notion that H2A.Z acts as a marker of active transcription throughout its distribution across the genome where it promotes and participates in the regulation of chromatin structure [31].
Increased binding of H2A.Z was detected in genes that were expressed at higher levels during ISCR (Figure 3). This suggests that the presence of $\mathrm{H} 2 \mathrm{~A} . \mathrm{Z}$ in the chromatin structure may assist in the recruitment of transcriptional machinery, which maybe not only aids exogenous transcriptional factors in binding to genes required for pluripotency but also maintains the epigenetic status of stem cells [32]. Previous data have revealed that a stress response occurs at the beginning of reprogramming process [8]. Here, we have found that $\mathrm{H} 2 \mathrm{~A} . \mathrm{Z}$ was enriched in genes involved in the biological processes of response to hypoxia, response to oxygen levels, response to steroid hormone stimulus, regulation of apoptosis, and regulation of cell death at the beginning of ISCR. Similarly, a particular set of occupied genes which only appeared in Day 7 cells were related to chromatin assembly. Our data showed that the total number of occupied genes in Day 7 cells was decreased and the number of genes occupied solely in these cells was the lowest (Figure 6(a)). However, the enrichment of H2A.Z in gene promoter regions in Day 7 cells was the highest (Figures 1(d) and 2(a)). Furthermore, the number of occupied genes present in both MEF and Day 7 cells was greater than the number present in both Day 7 cells and iPSCs. This indicates that H2A.Z tends to occupy a smaller and specialized subset of genes during reprogramming and that $\mathrm{H} 2 \mathrm{~A}$. $\mathrm{Z}$ related genes received the largest amount of enrichment at the promoter in Day 7 cells.

Reprogramming is a process involving changes to both gene expression and epigenetic modification. Previous data have shown that MEFs and Day 7 cells share a similar transcription profile [18], whereas the transcription profile of iPSCs is reversed and transcription factor overexpression 


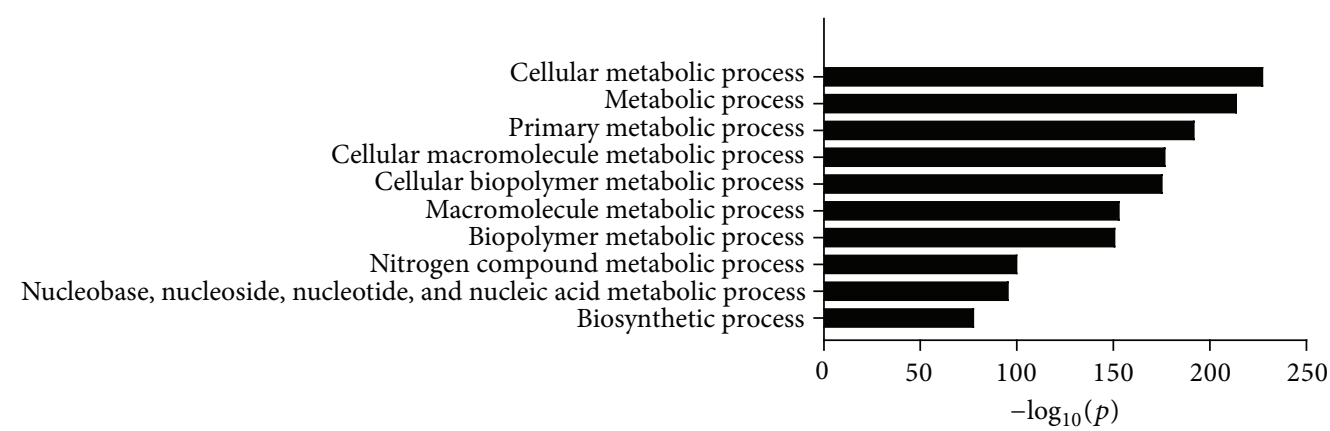

(a)

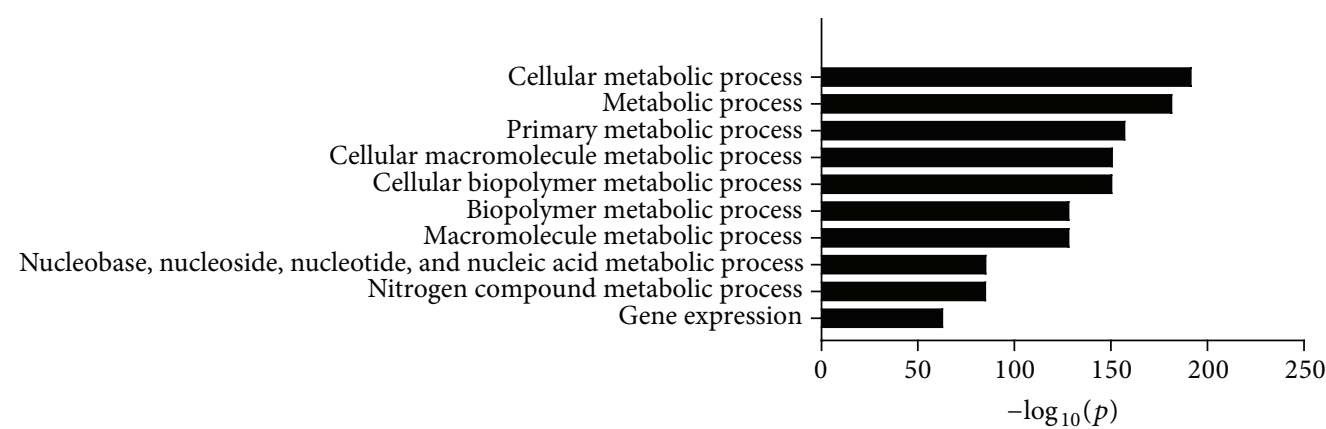

Day 7

(b)

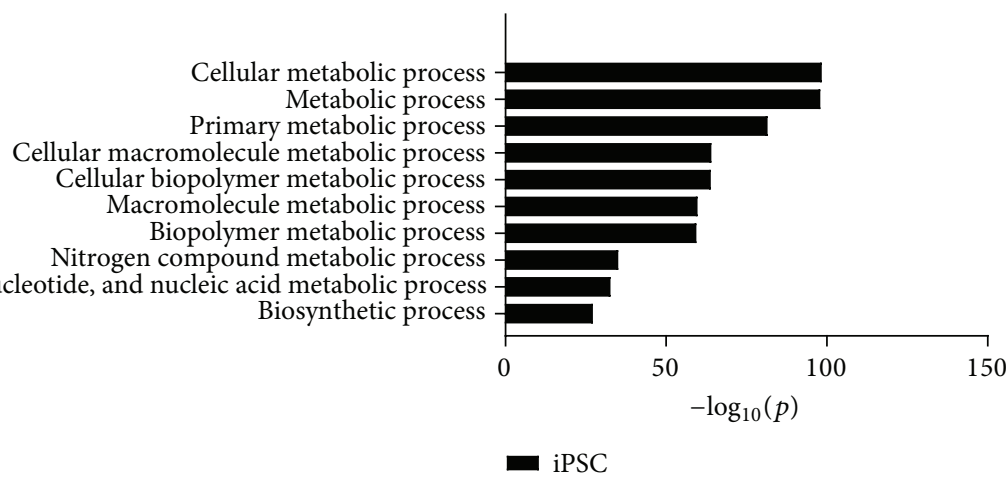

(c)

Figure 5: Top 10 biological processes undertaken by H2A.Z-associated genes in MEFs (a), Day 7 cells (b), and IPSCs (c). $p$ values for revaluation of $\mathrm{GO}$ categories among genes show evidence for enrichment of H2A.Z.

can favor the upregulation of epigenetic factor genes [18]. $\mathrm{H} 2 \mathrm{~A} . \mathrm{Z}$ can act as marks on open chromatin at promoters; it can recruit by PRC2/MLL complexes [33] or be recruited as OCT4 coactivators [23]. And histone acetylation may also act as active gene markers in genome-wide setting to recruit RNA polymerase II promoter [34] to reestablish gene expression pattern. As H2A.Z occupied p300-bound enhancers to support a global change of pluripotent genes [23], it showed that H2A.Z was predicted as the active markers for chromatin remodeling and supplied the accessible binding sites to epigenetic factors or transcriptional factors. We speculate that the enrichment of $\mathrm{H} 2 \mathrm{~A} . \mathrm{Z}$ in the promoter regions of a small number of specialized genes in Day 7 cells may represent the initiation of a changeover phase during reprogramming. Firstly, the enhanced incorporation of H2A.Z around specific genes' TSS in Day 7 cells can change chromatin structure on the local scale [31] and supplies an accessible binding site for epigenetic or transcription factors to exert their effects. And then, the expression of the specific genes has been changed which can alter chromatin structure of the whole genome (Figures 4 and 6(b)). At last, a large number of genes' expression changed to promote reprogramming, accompanied with incorporation in chromatin enrichment to fulfil epigenetic reprogramming (Figure S4). However, all of these predictions are merely a starting point for the study of H2A.Z in ISCR, and the exact functions are still waiting for more experimental evidence to be verified. 


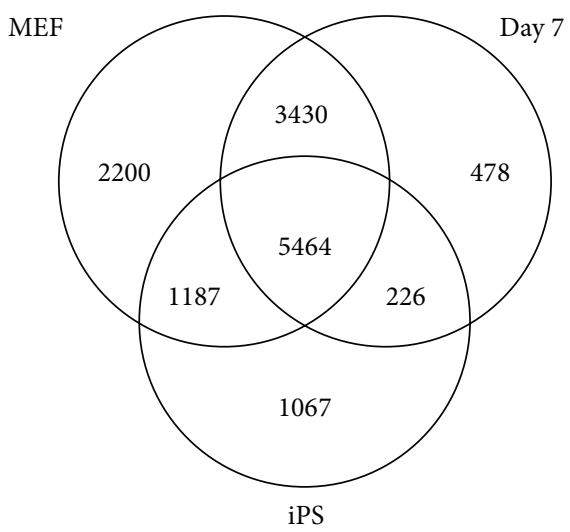

(a)

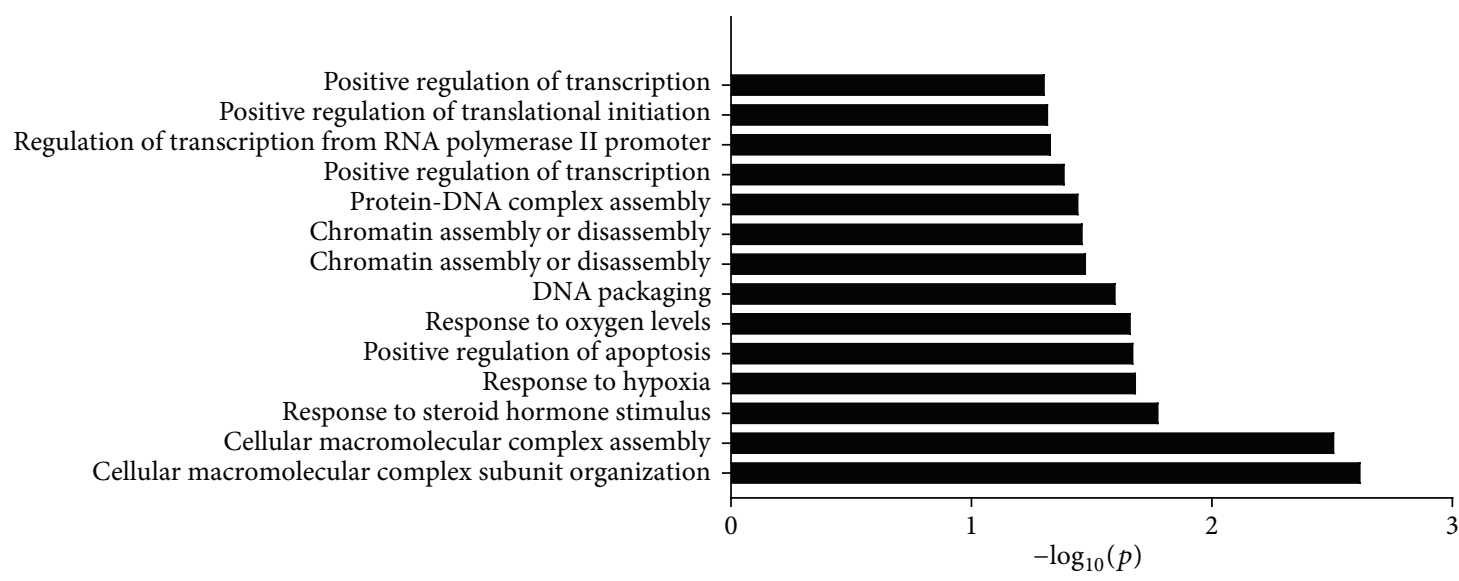

(b)

Figure 6: Distribution of H2A.Z during reprogramming and special functions of H2A.Z in Day 7 cells. (a) Venn diagram depiction of the number of genes occupied with H2A.Z in different cell samples. (b) Biological processes involved with the special genes occupied with H2A.Z in Day 7 cells.

\section{Materials and Methods}

4.1. Ethics Statement. Animal studies were carried out in a specific pathogen-free animal facility accredited by the Association for the Assessment and Accreditation of Laboratory Animal Care. All animal protocols were approved by the Animal Care and Use Committee of the Model Animal Research Center, the host for the National Resource Center for Mutant Mice in China, Nanjing University.

4.2. Cell Culture. C57BL6 MEFs were derived from embryonic day 13.5 mice embryos [35] and maintained in Dulbecco's modified Eagle's medium (DMEM, high glucose) containing $10 \%$ fetal bovine serum. ESCs and iPSCs were cultured on $\gamma$-irradiated MEFs in ES medium containing Knockout DMEM supplemented with 20\% Knockout Serum Replacement, $0.1 \mathrm{mM}$ NEAA, $2 \mathrm{mM}$ L-glutamine, $1 \mathrm{mM}$ sodium pyruvate, $0.1 \mathrm{mM} \beta$-mercaptoethanol, $50 \mathrm{U}$ and $50 \mathrm{mg} / \mathrm{mL}$ penicillin/streptomycin, and 1,000 U/mL leukemia inhibitory factor (Millipore, Billerica, MA, USA). All other reagents were purchased from Invitrogen Corp. (Carlsbad, CA, USA).

4.3. iPSC Generation. Plat-E cells were transfected at $80 \%$ confluence with PMX-based retroviral vectors (Addgene,
Cambridge, MA, USA) containing murine Oct4, Sox2, and Klf4 cDNAs using Lipofectamine 2000 (Invitrogen). Virus supernatant was harvested 48 hours after transfection. MEFs at passage 2 or 3 were seeded at a density of 3,5005,000 cells $/ \mathrm{cm}^{2}$ and incubated with filtered viral supernatants containing equal parts of the three transcription factors (Oct4, Sox2, and Klf4) [36] and $5 \mu \mathrm{g} / \mathrm{mL}$ polybrene. Twelve hours later, the medium was gradually replaced with ESC medium supplemented with $50 \mu \mathrm{g} / \mathrm{mL}$ Vc (Sigma-Aldrich, St. Louis, MO, USA) using a 4-day stepwise process [37, 38]. And all the induced cell clones were alkaline phosphatase (AP) positive and can develop to teratoma with three primary kind germ cell layers in vivo. In addition, the m-iPSC clones still keep a normal karyotype and can express typical pluripotent gene markers such as SSEA-1, Nanog, and Oct4 [18].

4.4. ChIP-Seq. Anti-H2A.Z antibody (mAb) was used for immunoprecipitation. Cells $\left(1 \times 10^{7}\right)$ were fixed with $1 \%$ fresh formaldehyde at $37^{\circ} \mathrm{C}$ for $10 \mathrm{~min}$ and lysed in SDS lysis buffer. The nuclei were resuspended and sonicated on ice to chromatin of $200-1000 \mathrm{bp}$ DNA. A total of $50 \mu \mathrm{L}$ of Dynal protein $\mathrm{G}$ beads, $5 \mu \mathrm{g}$ of antibody, and the sonicated chromatin were incubated at $4^{\circ} \mathrm{C}$ overnight. Precipitated 
immunocomplexes were treated with proteinase $\mathrm{K}$ at $65^{\circ} \mathrm{C}$ for $2 \mathrm{~h}$ and DNA was purified with Qiagen quick polymerase chain reaction (PCR) purification kit (Qiagen, Dusseldorf, Germany). ChIP DNA was amplified, ligated with adaptor, and re-paired at the DNA end. The $150 \mathrm{bp}$ DNA fragments were isolated from agar gel and sequenced with a Solexa Illumina $2 \mathrm{G}$ genetic analyzer.

4.5. ChIP-Seq Analysis. Solexa pipeline analysis was performed as described [39]. ChIP-Seq data were processed and aligned to the reference genome mm9. SOAP 2.21 software was used to map the 36 nucleotide sequence tags to the mouse genome with two mismatches allowed. Firstly, the raw data were obtained from the sequencing machine Illumina HiSeq2000, and clean data were extracted through quality control and filtering analysis process, and then the unique mapped reads were fulfilled with align analysis. MACS 1.4.0 software was used to identify the enriched peaks of the H2A.Z occupied sites. The program parameters were set as follows: $p$ value of $<10^{-4}$, model fold of 32 , and band width of $300 \mathrm{bp}$ for the mouse genome. Promoters were defined as $2 \mathrm{~kb}$ upstream all annotated TSSs and "downstream" refers to the region $2 \mathrm{~kb}$ downstream a TES site. Composite plots were generated by averaging values in each of the $200 \mathrm{bp}$ windows. Statistical significance of enrichment $\left(p<10^{-4}\right)$ was determined based on background distribution of randomized reads specific for each independent genome-wide ChIP analysis.

4.6. Gene Ontology. Gene ontology analysis was performed with DAVID (http://david.abcc.ncifcrf.gov/). EntrezGene IDs were used for the generation of enrichment statistics for the biological process category on the basis of a background list of all represented genes on the promoter microarray design.

\section{Conflict of Interests}

The authors declare that there is no conflict of interests regarding the publication of this paper.

\section{Authors' Contribution}

Fulu Dong, Zhenwei Song, and Jiali Yu are equal contributors.

\section{Acknowledgments}

The authors wish to thank Bin Bai (Shanghai Genergy Biotech. Co. Ltd.) for their technical assistance. This research was supported by Projects of Cultivating New Varieties by Transgenic Technology (no. 2008ZX08006-003), the Key Project of Chinese National Programs for Fundamental Research and Development (973 Program no. 2010CB945404), and National Natural Science Foundation of China to Fulu Dong (81300553).

\section{References}

[1] K. Takahashi and S. Yamanaka, "Induction of pluripotent stem cells from mouse embryonic and adult fibroblast cultures by defined factors," Cell, vol. 126, no. 4, pp. 663-676, 2006.
[2] K. Plath and W. E. Lowry, "Progress in understanding reprogramming to the induced pluripotent state," Nature Reviews Genetics, vol. 12, no. 4, pp. 253-265, 2011.

[3] M. C. Puri and A. Nagy, "Concise review: embryonic stem cells versus induced pluripotent stem cells: the game is on," Stem Cells, vol. 30, no. 1, pp. 10-14, 2012.

[4] C. Bönisch and S. B. Hake, "Histone H2A variants in nucleosomes and chromatin: more or less stable?", Nucleic Acids Research, vol. 40, no. 21, pp. 10719-10741, 2012.

[5] K. S. Sidhu, "New approaches for the generation of induced pluripotent stem cells," Expert Opinion on Biological Therapy, vol. 11, no. 5, pp. 569-579, 2011.

[6] M. Altaf, A. Auger, M. Covic, and J. Côté, "Connection between histone $\mathrm{H} 2 \mathrm{~A}$ variants and chromatin remodeling complexes," Biochemistry and Cell Biology, vol. 87, no. 1, pp. 35-50, 2009.

[7] Y. Buganim, D. A. Faddah, A. W. Cheng et al., "Single-cell expression analyses during cellular reprogramming reveal an early stochastic and a late hierarchic phase," Cell, vol. 150, no. 6, pp. 1209-1222, 2012.

[8] N. Mah, Y. Wang, M.-C. Liao et al., "Molecular insights into reprogramming-initiation events mediated by the OSKM gene regulatory network," PLoS ONE, vol. 6, no. 8, Article ID e24351, 2011.

[9] S. Pinato, C. Scandiuzzi, N. Arnaudo, E. Citterio, G. Gaudino, and L. Penengo, "RNF168, a new RING finger, MIU-containing protein that modifies chromatin by ubiquitination of histones H2A and H2AX," BMC Molecular Biology, vol. 10, article 55, 2009.

[10] Y. Wan, R. A. Saleem, A. V. Ratushny et al., "Role of the histone variant $\mathrm{H} 2 \mathrm{~A} . \mathrm{Z} / \mathrm{Htzlp}$ in TBP recruitment, chromatin dynamics, and regulated expression of oleate-responsive genes," Molecular and Cellular Biology, vol. 29, no. 9, pp. 2346-2358, 2009.

[11] E. Meshorer and T. Misteli, "Chromatin in pluripotent embryonic stem cells and differentiation," Nature Reviews Molecular Cell Biology, vol. 7, no. 7, pp. 540-546, 2006.

[12] E. Meshorer, D. Yellajoshula, E. George, P. J. Scambler, D. T. Brown, and T. Misteli, "Hyperdynamic plasticity of chromatin proteins in pluripotent embryonic stem cells," Developmental Cell, vol. 10, no. 1, pp. 105-116, 2006.

[13] L. Ho and G. R. Crabtree, "Chromatin remodelling during development," Nature, vol. 463, no. 7280, pp. 474-484, 2010.

[14] M. Shahhoseini, A. Taei, N. Z. Mehrjardi, G. H. Salekdeh, and H. Baharvand, "Epigenetic analysis of human embryonic carcinoma cells during retinoic acid-induced neural differentiation," Biochemistry and Cell Biology, vol. 88, no. 3, pp. 527-538, 2010.

[15] R. K. Suto, M. J. Clarkson, D. J. Tremethick, and K. Luger, "Crystal structure of a nucleosome core particle containing the variant histone H2A.Z," Nature Structural Biology, vol. 7, no. 12, pp. 1121-1124, 2000.

[16] V. Subramanian, P. A. Fields, and L. A. Boyer, "H2A.Z: a molecular rheostat for transcriptional control," F1000Prime Reports, vol. 7, article 01, 2015.

[17] N. Takakura, "Formation and regulation of the cancer stem cell niche," Cancer Science, vol. 103, no. 7, pp. 1177-1181, 2012.

[18] F. Dong, Z. Song, J. Zhang et al., "Global transcriptional analysis of nuclear reprogramming in the transition from MEFs to iPSCs," Genes to Cells, vol. 18, no. 1, pp. 42-55, 2013.

[19] Q. Zhu, G. Wani, H. H. Arab, M. A. El-Mahdy, A. Ray, and A. A. Wani, "Chromatin restoration following nucleotide excision repair involves the incorporation of ubiquitinated $\mathrm{H} 2 \mathrm{~A}$ at damaged genomic sites," DNA Repair, vol. 8, no. 2, pp. 262-273, 2009. 
[20] Y. Zhang, T. Liu, C. A. Meyer et al., "Model-based analysis of ChIP-Seq (MACS)," Genome Biology, vol. 9, no. 9, article R137, 2008.

[21] M. L. Conerly, S. S. Teves, D. Diolaiti, M. Ulrich, R. N. Eisenman, and S. Henikoff, "Changes in H2A.Z occupancy and DNA methylation during B-cell lymphomagenesis," Genome Research, vol. 20, no. 10, pp. 1383-1390, 2010.

[22] K. Kim, V. Punj, J. Choi et al., "Gene dysregulation by histone variant H2A.Z in bladder cancer," Epigenetics and Chromatin, vol. 6, article 34, 2013.

[23] G. Hu, K. Cui, D. Northrup et al., "H2A.Z facilitates access of active and repressive complexes to chromatin in embryonic stem cell self-renewal and differentiation," Cell Stem Cell, vol. 12, no. 2, pp. 180-192, 2013.

[24] A. Barski, S. Cuddapah, K. Cui et al., "High-resolution profiling of histone methylations in the human genome," Cell, vol. 129, no. 4, pp. 823-837, 2007.

[25] P. Billon and J. Côté, "Precise deposition of histone H2A.Z in chromatin for genome expression and maintenance," Biochimica et Biophysica Acta-Gene Regulatory Mechanisms, vol. 1819, no. 3-4, pp. 290-302, 2012.

[26] K. Bruce, F. A. Myers, E. Mantouvalou et al., "The replacement histone H2A.Z in a hyperacetylated form is a feature of active genes in the chicken," Nucleic Acids Research, vol. 33, no. 17, pp. 5633-5639, 2005.

[27] C. Jin, C. Zang, G. Wei et al., "H3.3/H2A.Z double variantcontaining nucleosomes mark "nucleosome-free regions" of active promoters and other regulatory regions," Nature Genetics, vol. 41, no. 8, pp. 941-945, 2009.

[28] H. Zhang, D. N. Roberts, and B. R. Cairns, "Genome-wide dynamics of Htzl, a histone H2A variant that poises repressed/ basal promoters for activation through histone loss," Cell, vol. 123, no. 2, pp. 219-231, 2005.

[29] R. Karnik and A. Meissner, "Browsing (Epi)genomes: a guide to data resources and epigenome browsers for stem cell researchers," Cell Stem Cell, vol. 13, no. 1, pp. 14-21, 2013.

[30] A. Peserico and C. Simone, "Physical and functional HAT/HDAC interplay regulates protein acetylation balance," Journal of Biomedicine and Biotechnology, vol. 2011, Article ID 371832, 10 pages, 2011.

[31] M. Altaf, A. Auger, M. Covic, and J. Côté, "Connection between histone H2A variants and chromatin remodeling complexes," Biochemistry and Cell Biology, vol. 87, no. 1, pp. 35-50, 2009.

[32] A. Gaspar-Maia, Z. A. Qadeer, D. Hasson et al., "MacroH2A histone variants act as a barrier upon reprogramming towards pluripotency," Nature Communications, vol. 4, article 1565, 2013.

[33] Y. Shiozawa, E. A. Pedersen, A. M. Havens et al., "Human prostate cancer metastases target the hematopoietic stem cell niche to establish footholds in mouse bone marrow," The Journal of Clinical Investigation, vol. 121, no. 4, pp. 1298-1312, 2011.

[34] M. P. Creyghton, A. W. Cheng, G. G. Welstead et al., "Histone H3K27ac separates active from poised enhancers and predicts developmental state," Proceedings of the National Academy of Sciences of the United States of America, vol. 107, no. 50, pp. 21931-21936, 2010.

[35] D. A. Conner, "Mouse embryo fibroblast (MEF) feeder cell preparation," Current Protocols in Molecular Biology, chapter 23: unit 23.2, 2001.

[36] K. Takahashi, K. Okita, M. Nakagawa, and S. Yamanaka, "Induction of pluripotent stem cells from fibroblast cultures," Nature Protocols, vol. 2, no. 12, pp. 3081-3089, 2007.
[37] J. Chen, J. Liu, Q. Han et al., “Towards an optimized culture medium for the generation of mouse induced pluripotent stem cells," Journal of Biological Chemistry, vol. 285, no. 40, pp. 3106631072, 2010.

[38] M. A. Esteban, T. Wang, B. Qin et al., "Vitamin C enhances the generation of mouse and human induced pluripotent stem cells," Cell Stem Cell, vol. 6, no. 1, pp. 71-79, 2010.

[39] A. Valouev, D. S. Johnson, A. Sundquist et al., "Genome-wide analysis of transcription factor binding sites based on ChIP-Seq data," Nature Methods, vol. 5, no. 9, pp. 829-834, 2008. 

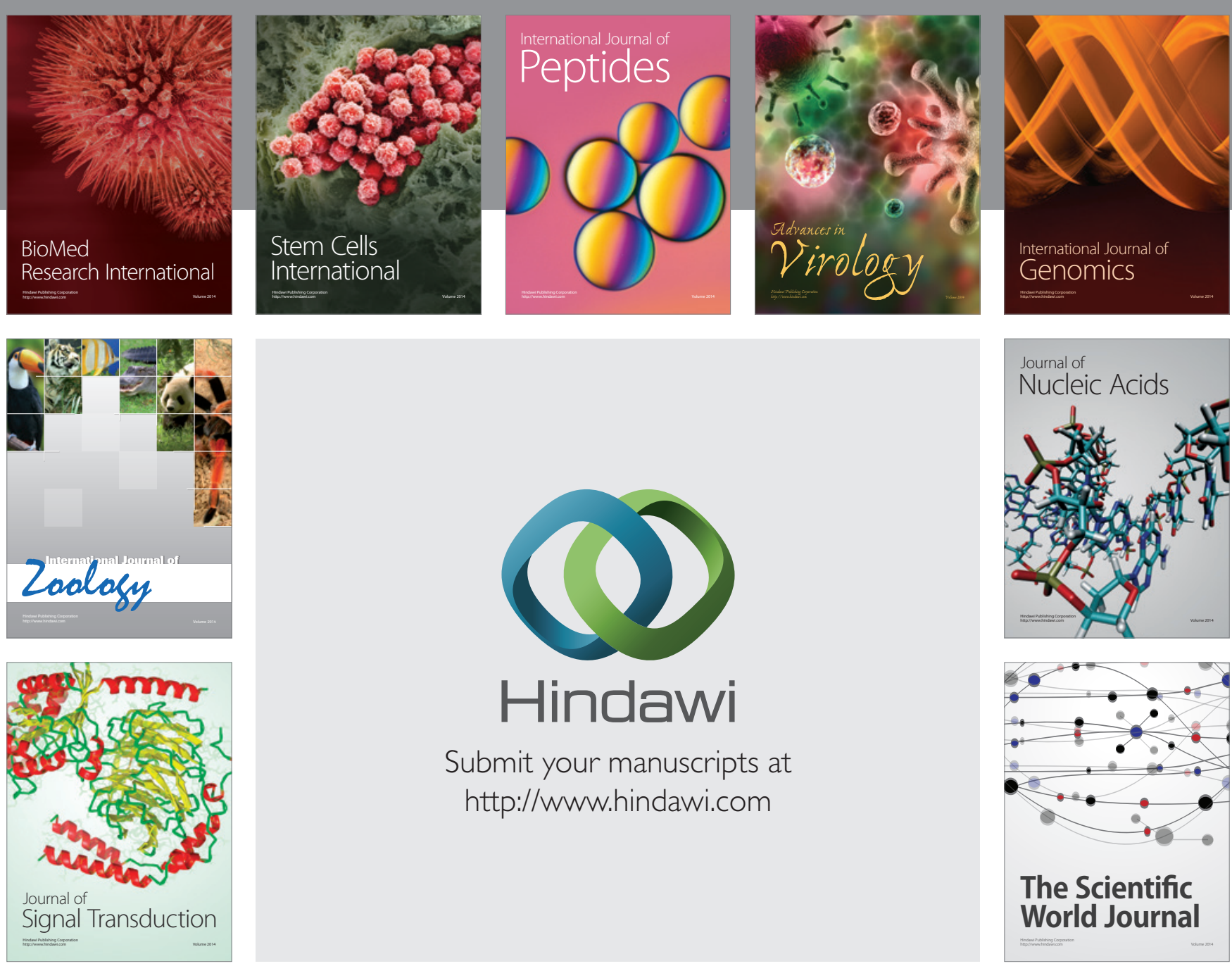

Submit your manuscripts at

http://www.hindawi.com
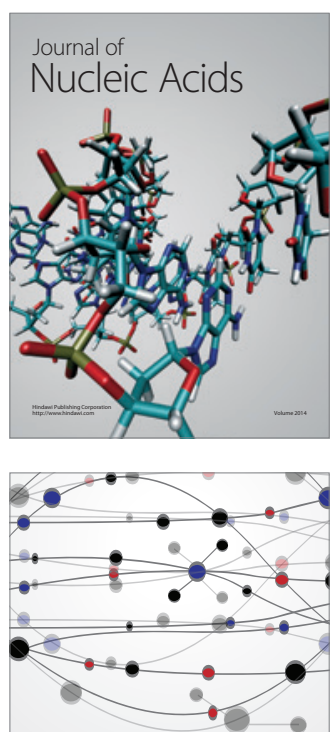

The Scientific World Journal
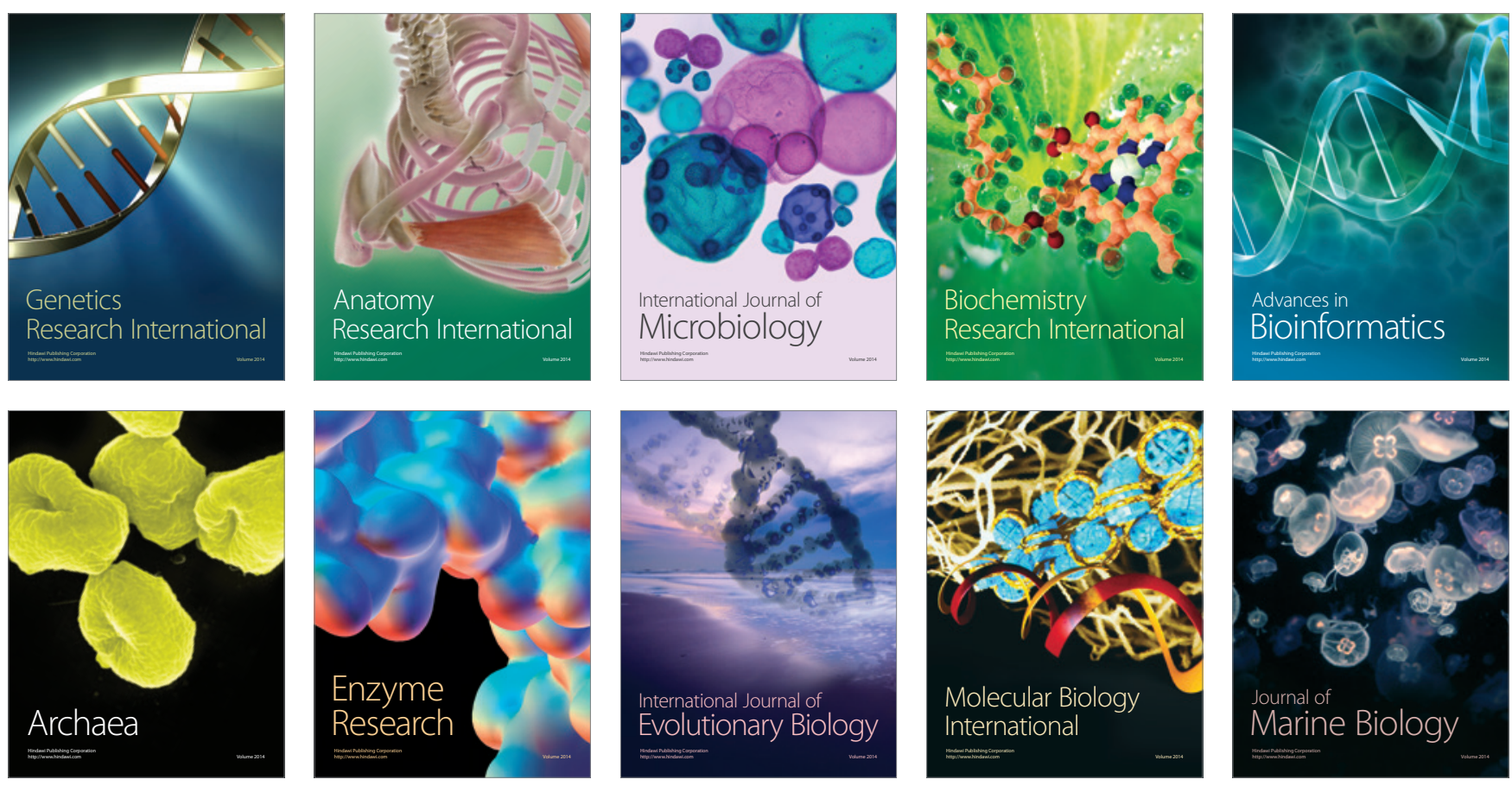